\title{
Recurrent Aortitis Associated With IgG4-Related Disease
}

Antonio Lalueza, MD, PhD; Sebastián Ruiz, MD, PhD; Felipe Villacampa, MD; Rocío López, MD;

Elena Zabia, MD; Pilar Sarandeses, MD; Ana Cristina Hernández, MD; Juan María Herrero, MD; Carlos Lumbreras, MD, PhD

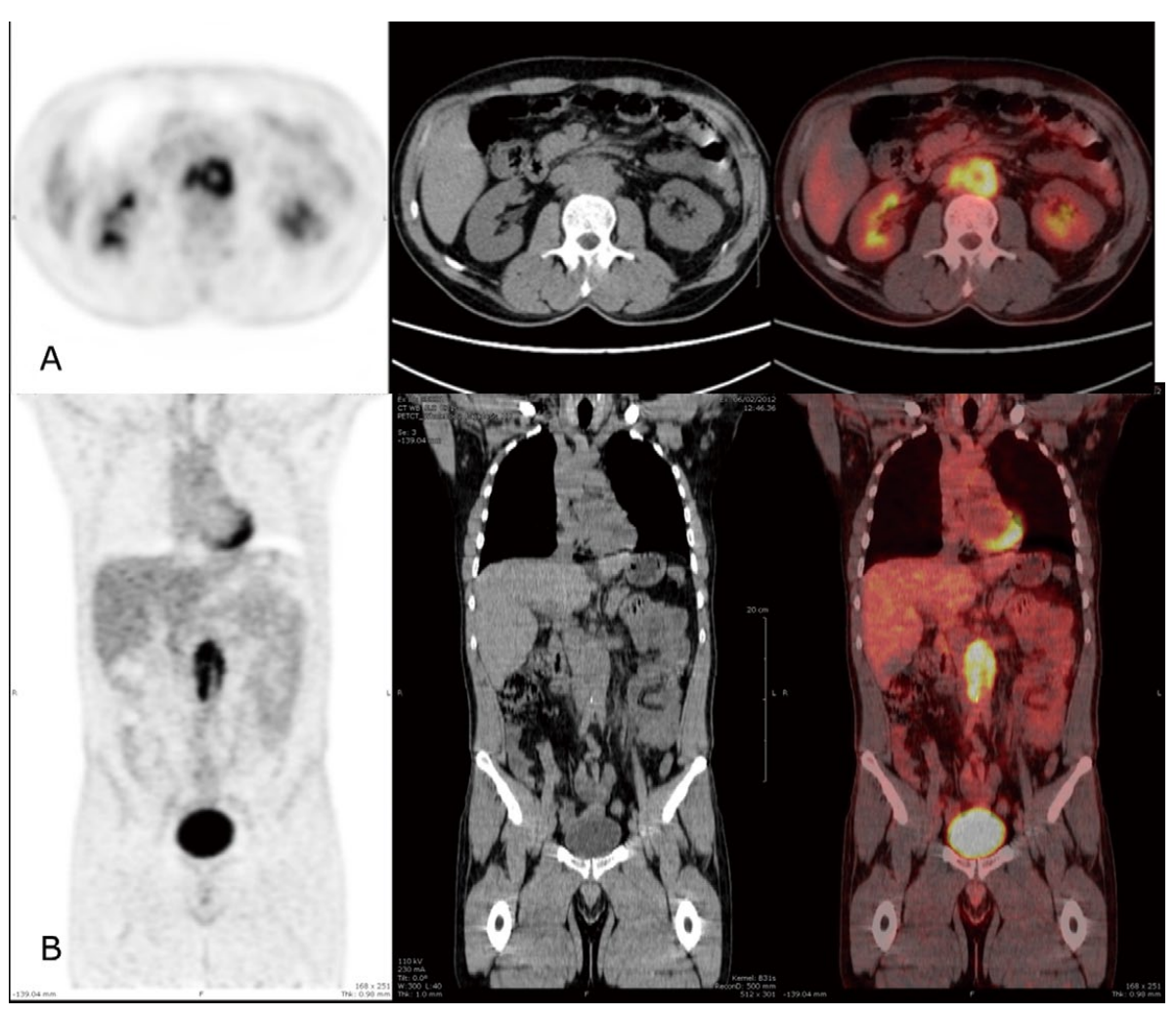

Figure 1. First fluorine-18 fluorodeoxyglucose (FDG) positron emission tomography/computed tomography (FDG-PET/CT). (A) Axial PET, CT and fused PET/CT and (B) corresponding coronal imaging showing peri-aortic soft-tissue mantle in the abdominal aorta with FDG uptake from the renal arteries to the iliac bifurcation $(2.9 \times 2.9 \times 7.6 \mathrm{~cm})$ with maximum standardized uptake value of 12.55 .

$\mathbf{V}$ ascular involvement as the clinical presentation of IgG4-related disease (IgG4-RD) is rare. Here, we report a case of aortic involvement with recurrence after initial response to steroids, subsequently treated with azathioprine. To our knowledge, this is the first case of recur- rent IgG4-RD aortitis successfully treated with azathioprine.

The patient was a 46-year-old man with a history of nonmetastatic clear-cell renal carcinoma (pT1aNOM0) treated with right radical nephrectomy 5 months previously. On admission, he reported progressive lumbar pain, night sweats, fatigue

Received November 10, 2015; revised manuscript received January 8, 2016; accepted January 24, 2016; released online February 25, 2016 Time for primary review: 37 days

Department of Internal Medicine (A.L., J.M.H., C.L.), Department of Nuclear Medicine (S.R., P.S., A.C.H.), Department of Urology (F.V.), Department of Pathology (R.L.), Department of Radiology (E.Z.), Hospital 12 de Octubre, Madrid; Department of Medicine (A.L., C.L.), Department of Radiology and Nuclear Medicine (S.R.), School of Medicine, Complutense University, Madrid; and Research Institute 12 de Octubre (i+12), Madrid (C.L.), Spain

Mailing address: Antonio Lalueza Blanco, MD, PhD, Department of Internal Medicine, University Hospital 12 de Octubre, Av. Córdoba km 5.400, 28041 Madrid, Spain. E-mail: lalueza@hotmail.com

ISSN-1346-9843 doi:10.1253/circj.CJ-15-1186

All rights are reserved to the Japanese Circulation Society. For permissions, please e-mail: cj@j-circ.or.jp 

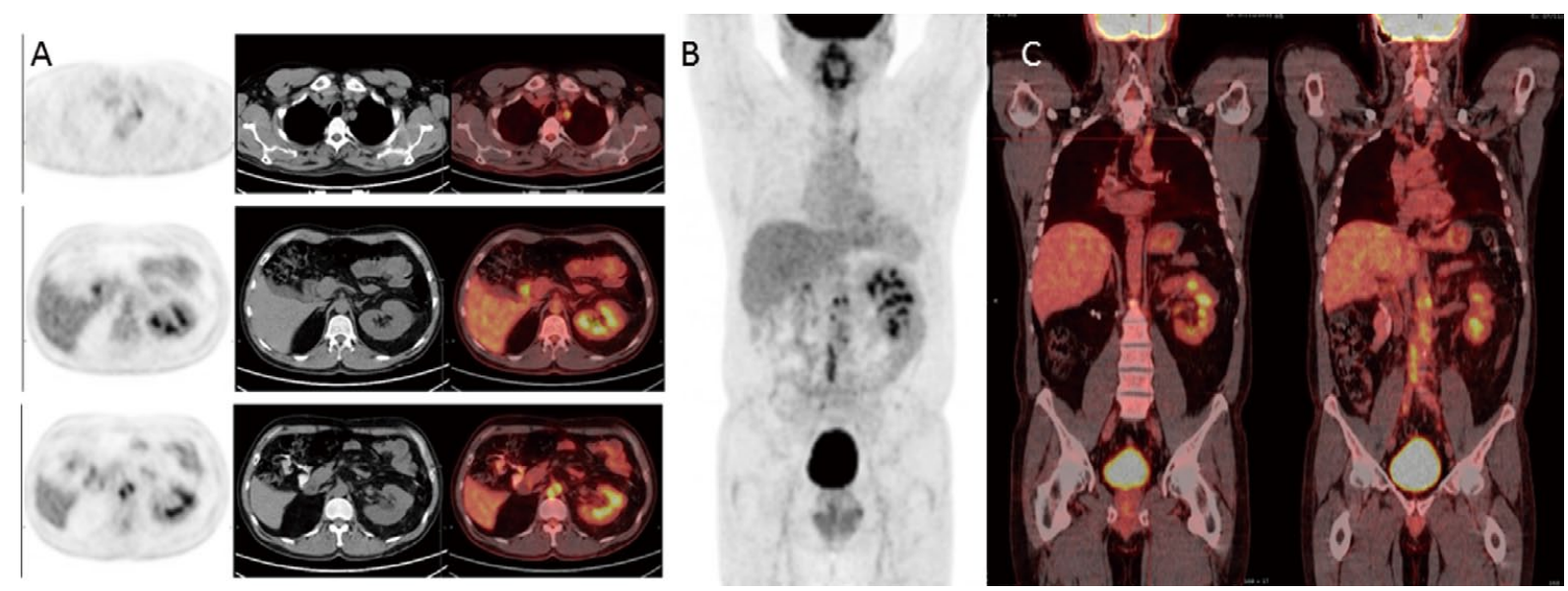

Figure 2. After completion of steroid treatment, further fluorine-18 fluorodeoxyglucose (FDG) positron emission tomography/ computed tomography (FDG-PET/CT) was performed. (A) Axial PET, CT and fused PET/CT, (B) maximum-intensity-projection PET and (C) coronal fused PET/CT showed a new increased FDG uptake localized in the proximal left subclavian artery, and increased periaortic activity. Furthermore, new tracer uptake was observed in the second portion of the duodenum, close to the ampulla of Vater.

and weight loss. Physical examination was normal except for the presence of cervical lymphadenopathy. Computed tomography (CT) indicated an adenopathic conglomerate surrounding the internal jugular vein without contrast enhancement, and mildly enlarged $(<1 \mathrm{~cm})$ enhanced mediastinal and axillary lymph nodes. Additionally, abdominal CT showed a softtissue-density mass surrounding the abdominal aorta from the renal arteries to the aortic bifurcation including left renal vein and inferior vena cava, suggestive of retroperitoneal fibrosis. Laboratory results were normal except for an accelerated erythrocyte sedimentation rate (ESR; $43 \mathrm{~mm} / \mathrm{h})$. HIV serology, auto-antibodies and tumor markers were negative. Fluorine-18 fluorodeoxyglucose (FDG) positron emission tomography-CT (FDG-PET/CT) showed marked radiotracer enhancement at the cervical adenopathic conglomerate with a maximum standardized uptake value (SUV $\max$ ) of 17.63. Metabolically significant thoracic lymph nodes were not appreciated. Abdominal imaging showed an extensive hypermetabolic soft-tissue density surrounding the abdominal aorta from the renal arteries to the iliac artery bifurcation with an $\mathrm{SUV}_{\max }$ of 12.55 , as well as interaortocaval and left para-aortic lymphadenopathies (Figure 1). Cervical lymph node biopsy was performed. There was no evidence of neoplastic cells and the immunohistochemistry was negative for epithelial cells. Serum immunoglobulins were normal: total IgG, $769 \mathrm{mg} / \mathrm{dl} ; \mathrm{IgG} 4,36 \mathrm{mg} / \mathrm{dl}$ (reference range, 3-210). Given the concomitant retroperitoneal involvement and the negative result of the previous biopsy, specific immunohistochemistry for IgG4 staining of the lymph node was performed, showing numerous IgG4-positive plasma cells. The mean proportion of IgG4/total IgG-positive cells was $56.5 \%$, compatible with the diagnosis of IgG4-RD (Figure S1). Prednisone was initiated at $0.8 \mathrm{mg} / \mathrm{kg} /$ day for 4 weeks, then tapered over a period of 8 months, to zero. FDG-PET/CT at 6 months (Figure S2) showed metabolic normalization of the lymphadenopathies and of the retroperitoneal activity but showed substantial accumulation of FDG in the right common iliac artery. Ten months later, the patient underwent new FDG-PET/CT (Figure 2) in which we observed that previous enlarged lymph nodes remained stable in size but a new FDG uptake appeared in the proximal left subclavian artery and the abdominal periaortic activity had also increased. In the light of these results, recurrence of IgG4-RD was assumed, and azathioprine treatment was started at a dose of $2 \mathrm{mg} / \mathrm{kg}$ body weight/day. FDG-PET/CT after 8 months of azathioprine, showed complete normalization of abnormal FDG uptake seen on the previous PET.

IgG4-RD was first described in 2001 and recognized as a systemic disease in 2003. IgG4-RD is considered a rare entity that can involve virtually any anatomical structure, although vascular involvement is unusual.1,2 Previous studies have shown that $10 \%$ of inflammatory thoracic aortitis $(75 \%$ of cases of lymphoplasmacytic aortitis) and between $1 \%$ and $6 \%$ of all abdominal aortic aneurysms are associated with IgG4-RD. ${ }^{3}$ In the advent of cardiovascular presentation of IgG4-RD, abdominal aorta is more commonly affected but it rarely develops in other locations. ${ }^{4-6}$

Some important points from the present case are worth mentioning. Diagnosis and close monitoring on FDG-PET-/CT enabled early diagnosis of arterial involvement, which may have avoided subsequent cardiovascular complications such as aneurysm or aortic dissection. ${ }^{3,7}$ In contrast, the patient had an IgG4-RD relapse with subclavian artery involvement after finishing steroid treatment, illustrating two important clinical events: (1) recurrence occurred in a different location from the initial arterial involvement, the subclavian artery, which, furthermore, has been seldom described in IgG4-RD; and (2) the recurrence had a different clinical presentation: at the time of diagnosis, periarterial inflammatory involvement predominated, while the recurrence emerged as a large vessel arteritis.

Available data support the efficacy of steroids in the treatment of peri-aortic sclerosing inflammation, but the steroid response rate is not well known, although in the pancreatic form of the disease it has reached $89 \% .{ }^{8}$ Although steroid therapy could, in theory, increase the risk of aneurysmal rupture by making the wall thinner and more fragile, no significant changes in diameter of the aortic aneurysm after steroid therapy have 
been noted..$^{3,9}$

Previously, no studies had examined the risk factors associated with relapse after steroid therapy in IgG4-RD cardiovascular disorders. ${ }^{10}$ The relapse rates in other clinical forms, mainly pancreatic, have been estimated at $32 \%$ after 6 months of treatment and $56 \%$ after 1 year. $^{8-10}$ For patients with recurrent IgG4-RD, long-term treatment strategies with either azathioprine at a dose of $2-2.5 \mathrm{mg} / \mathrm{kg}$ body weight/day, or mycophenolate mofetil at a dose of $750 \mathrm{mg}$ twice daily, appear to be useful. ${ }^{8,11,12}$ Finally, we note that, to the best of our knowledge, reports on recurrence of cardiovascular involvement of IgG4-RD successfully treated with azathioprine are rare. ${ }^{13}$ In the present case, the patient had sustained clinical response as verified on sequential FDG-PET/CT.

\section{Acknowledgments}

Sincere thanks to Alejandra Morales-Cartagena, Department of Internal Medicine, University Hospital 12 de Octubre, for review of the manuscript.

\section{Disclosures}

None.

\section{References}

1. Inoue $\mathrm{D}$, Zen $\mathrm{Y}$, Abo H, Gabata T, Demachi H, Yoshikawa J, et al. Immunoglobulin G4-related periaortitis and periarteritis: CT findings in 17 patients. Radiology 2011; 261: 625-633.

2. Kasashima S, Zen Y, Kawashima A, Endo M, Matsumoto Y, Kasashima $\mathrm{F}$, et al. A clinicopathologic study of immunoglobulin G4-related sclerosing disease of the thoracic aorta. J Vasc Surg 2010; 52: 1587 1595.

3. Stone JR. Aortitis, periaortitis, and retroperitoneal fibrosis, as manifestations of IgG4-related systemic disease. Curr Opin Rheumatol 2011; 23: 88-94.

4. Bito Y, Sasaki Y, Hirai H, Hosono M, Nakahira A, Suehiro Y, et al. A surgical case of expanding bilateral coronary aneurysms regarded as immunoglobulin G4-related disease. Circulation 2014; 129: e453-e456, doi:10.1161/CIRCULATIONAHA.114.008706.
5. Kasashima S, Zen Y. IgG4-related inflammatory abdominal aortic aneurysm. Curr Opin Rheumatol 2011; 23: 18-23.

6. Kouzu H, Miki T, Mizuno M, Kimura Y, Muranaka A, Yuda S, et al. Inflammatory myofibroblastic tumor of the heart: Overlap with IgG4-related disease? Circ J 2014; 78: 1006-1008.

7. Miyagawa M, Yokoyama R, Nishiyama Y, Ogimoto A, Higaki J, Mochizuki T. Positron emission tomography-computed tomography for imaging of inflammatory cardiovascular diseases: Sarcoidosis, large-vessel arteritis, and atherosclerosis. Circ J 2014; 78: $1302-$ 1310.

8. Kamisawa T, Shimosegawa T, Okazaki K, Nishino T, Watanabe H, Kanno A, et al. Standard steroid treatment for autoimmune pancreatitis. Gut 2009; 58: 1504-1507.

9. Mizushima I, Inoue D, Yamamoto M, Yamada K, Saeki T, Ubara Y, et al. Clinical course after corticosteroid therapy in IgG4-related aortitis/periaortitis and periarteritis: A retrospective multicenter study. Arthritis Res Ther 2014; 16: R156.

10. Tajima M, Nagai R, Hiroi Y. IgG4-related cardiovascular disorders. Int Heart J 2014; 55: 287-295.

11. Naitoh I, Nakazawa T, Ohara H, Sano H, Ando T, Hayashi K, et al. Autoimmune pancreatitis associated with various extrapancreatic lesions during a long-term clinical course successfully treated with azathioprine and corticosteroid maintenance therapy. Intern Med 2009; 48: 2003-2007.

12. Frustaci A, Chimenti C. Immunosuppressive therapy in myocarditis. Circ J 2015; 79: 4-7.

13. Loffler C, Hoffend J, Rebel M, Waldherr R, Uppenkamp M, Bergner $\mathrm{R}$. A rare cause for lower back pain: A case of an IgG4-related periaortitis. Clin Rheumatol 2016; 35: 265-270.

\section{Supplementary Files}

Supplementary File 1

Figure S1. Histological findings of the lymph node biopsy showing (A) dense lymphoplasmacytic infiltration, an obliterative phlebitis and storiform fibrosis (H\&E staining), and (B) infiltration of IgG4positive plasma cells (IgG4 immunostaining).

Figure S2. (A) Axial positron emission tomography (PET), computed tomography (CT) and fused PET/CT, and (B) corresponding coronal imaging.

Please find supplementary file(s);

http://dx.doi.org/10.1253/circj.CJ-15-1186 